\title{
Numerical simulation of endocytosis: Viscous flow driven by membranes with non-uniformly distributed curvature-inducing molecules
}

\author{
John Lowengrub ${ }^{\mathrm{b}, \mathrm{e}, \mathrm{c}}$, Jun Allard ${ }^{\mathrm{b}, \mathrm{d}, \mathrm{c}}$, Sebastian Aland ${ }^{\mathrm{a}, \mathrm{b}, \mathrm{c}}$ \\ ${ }^{a}$ Institut für wissenschaftliches Rechnen, TU Dresden, 01062 Dresden, Germany \\ ${ }^{b}$ Department of Mathematics, UC Irvine, Irvine, CA 92697, USA \\ ${ }^{c}$ Center for Complex Biological Systems, UC Irvine, CA 92697, USA \\ ${ }^{d}$ Department of Physics and Astronomy, UC Irvine, Irvine, CA 92697, USA \\ ${ }^{e}$ Department of Biomedical Engineering, UC Irvine, Irvine, CA 92697, USA
}

\begin{abstract}
The formation of membrane vesicles from a larger membrane that occurs during endocytosis and other cell processes are typically orchestrated by curvatureinducing molecules attached to the membrane. Recent reports demonstrate that vesicles can form de novo in a few milliseconds. Membrane dynamics at these scales are strongly influenced by hydrodynamic interactions. To study this problem, we develop new diffuse interface models for the dynamics of inextensible vesicles in a viscous fluid with stiff, curvature-inducing molecules. The model couples the Navier-Stokes equations with membrane-induced bending forces that incorporate concentration-dependent bending stiffness coefficients and spontaneous curvatures, with equations for molecule transport and for a Lagrange multiplier to enforce local inextensibility. Two forms of surface transport equations are considered: Fickian surface diffusion and Cahn-Hilliard surface dynamics, with the former being more appropriate for small molecules and the latter being better for large molecules. The system is solved using adaptive finite element methods in 3D axisymmetric geometries. The results demonstrate that hydrodynamics can indeed enable the rapid formation of a small vesicle attached to the membrane by a narrow neck. When the Fickian model is used, this is a transient state with the steady state being a flat membrane with a uniformly distributed molecule concentration due to diffusion. When the Cahn-Hilliard model is used, molecule concentration gradients are sustained, the neck stabilizes and the system evolves to a steady-state with a small, compact vesicle attached to the membrane. By varying the membrane coverage of molecules in the Cahn-Hilliard model, we find that there is a critical (smallest) neck radius and a critical (fastest) budding time. These critical points are associated with changes in the vesicle morphology from spherical to mushroom-like as the molecule coverage on the membrane is increased.
\end{abstract}

Email addresses: lowengrb@math.uci.edu (John Lowengrub), jun.allard@uci.edu (Jun Allard), sebastian.aland@tu-dresden.de (Sebastian Aland)

Preprint submitted to Elsevier

September 22, 2015

(C) 2015. This manuscript version is made available under the Elsevier user license http://www.elsevier.com/open-access/userlicense/1.0/ 
Keywords: Endocytosis, Navier-Stokes flow, Helfrich energy, Phase-field model, Clathrin, Numerical Simulation

\section{Introduction}

The biological membranes that surround cells and organelles often undergo shape changes as part of cellular processes. In one class of processes, which includes endocytosis, inter-organelle transport and virus entry [24, a small membrane vesicle is formed from a larger membrane. In others, locally curved membranes enable other mechanical processes such as cytoskeletal protrusion 30. These events are typically orchestrated by curvature-inducing molecules that dynamically attach to the membrane, such as clathrin and bar-domain proteins.

Classical clathrin-mediated endocytosis, which occurs on a timescale of seconds, has been extensively studied, including contributions from mathematical models [4, 25, 29] . However, recent reports demonstrate that in some circumstances vesicles can form de novo in a few milliseconds [41. Examples include ultrafast endocytosis at the neurological synapse, in which rapid endocytosis is necessary to complement rapid exocytosis of neurotransmitters 32 .

Ultrafast vesicle formation raises a fundamental biophysical question: Membrane dynamics at these scales (millisecond, nanometer) are dominated by hydrodynamic interactions, as the membrane pushes the intracellular and extracellular fluids around to accommodate curvature. What limits do hydrodynamic interactions impose on the speed of endocytosis? How do the resulting intracellular flows affect the arrival rate of soluble curvature-inducing molecules? What are the intermediate dynamic shapes of the membrane and what spatial constraints do these shapes place on membrane-associated molecules, such as clathrin and receptor cargo?

To address these questions, we have developed computational fluid dynamic models of a membrane interacting with intracellular and extracellular fluids. The identity of molecular participants in ultrafast endocytosis remains unknown. Therefore, we consider an abstract curvature-inducing molecule (henceforth CIM) and explore the dependence of vesicle formation on its properties. Known CIMs include proteins such as clathrin, caveolin, COP proteins, BAR domain proteins such as GRAF1 and epsins, as well as lipid modifiers 28. These range in size-per-molecule from $4 \mathrm{~nm}$ for epsin [21 to $50 \mathrm{~nm}$ for clathrin [16], and range in spontaneous curvature from zero to $9^{-1} \mathrm{~nm}^{-1}$ for synaptotagmin [28. For recent reviews of CIMs and other modes of biological membrane sculpting, see $23,28,33,36$. For simulation results, we use parameter values in the range of these known CIMs.

Mathematical models have been developed, and numerical simulations have been performed, for vesicles with variable biophysical properties due to the presence of multiple lipid components and embedded proteins using discrete and continuum approaches. See, for example, the review by Elson et al. [14. Here, we use a continuum phase field approach and extend our previous work for locally 


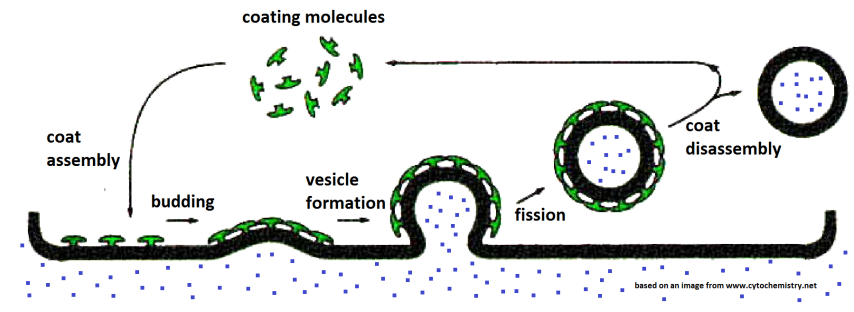

Figure 1: Schematic of the processes involved in endocytosis. Curvature inducing molecules attach to the membrane and induce out-of-plane deformations. Once enough molecules cover the membrane a vesicle is formed that provides the vehicle to transport extracellular cargo into the cell.

inextensible, homogeneous closed vesicles [2] to locally inextensible, heterogeneous membranes with CIMs. Although phase field models for heterogeneous vesicles have been developed previously, e.g., 40, 26, 18, 12, 44, 17, 15, 19, none of these approaches considered the effect of fluid flow (and local inextensibility). The effect of flow on the dynamics of locally inextensible, multicomponent vesicles was investigated using a combined boundary integral and surface phase field approach in 2D [35] and in 3D axisymmetric geometries [34. However to simulate endocytosis, which typically occurs on length scales of nanometers while the overall membrane may be millimeters in length, as in the experiments described above, only a part of membrane can be considered due to computational cost. This geometry is straightforward to implement using the phase field approach developed here. Further, the phase field model can also be extended to incorporate additional physical processes such as adsorption and desorption of CIMs following [37, 38, and is independent of dimension, which makes the extension to fully three dimensional geometries straightforward as well.

The outline of the paper is as follows. In Sec. 2 the mathematical model is presented. In Sec. 3 the discretization of the equations and the numerical methods used to solve the discrete system are discussed. In Sec. 4 , simulations demonstrating the convergence of the algorithm are presented along with an investigation of the influence of hydrodynamics and the dynamics of CIMs on the results. Finally, in Sec. 5 conclusions are drawn and future directions are discussed. Several technical results are derived in the Appendices.

\section{Mathematical Model}

Consider a membrane that is partially covered with molecules that have a non-zero bending modulus. Let us denote the membrane with $\Gamma$ and the covered part as $\Gamma_{c} \subset \Gamma$. Furthermore we assume that the spontaneous curvature is zero for a clean membrane and is $H_{0}$ for a membrane molecule. Then the total 


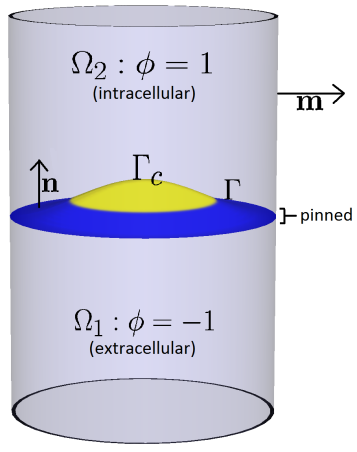

Figure 2: The cylindrical configuration used in the numerical simulations. The membrane $\Gamma$ is defined as the zero-level set of the phase field $\phi$ and separates the intracellular from the extracellular fluid region where $\phi=1$ or $\phi=-1$, respectively. A part of the membrane, $\Gamma_{c}$, is covered with CIM.

bending energy is

$$
E=\int_{\Gamma_{c}} \frac{1}{2} b_{c}\left(H-H_{0}\right)^{2} \mathrm{~d} A+\int_{\Gamma} \frac{1}{2} b_{m} H^{2} \mathrm{~d} A,
$$

where $b_{c}$ and $b_{m}$ are the bending moduli of the molecule and the clean membrane, respectively.

To keep track of $\Gamma_{c}$ it is convenient to introduce the non-dimensional molecule concentration $c(\mathbf{x})$. Let us assume $c$ to be normalized such that $c=1$ corresponds to complete coverage of the membrane. Therefore, $c$ may either be a coarse grained quantity denoting the normalized number of membrane molecules per surface area. Alternatively, $c$ might assume only discrete values: either 0 or 1 , indicating the presence or absence of a molecule at a certain location on the membrane. The latter use of $c$ would be particularly appropriate for large membrane molecules such as clathrin, whose size prevents the reasonable use of a coarse-grained concentration.

No matter which interpretation of $c$ is used, the energy of a CIM-covered membrane can be written generally as

$$
E=\int_{\Gamma} \frac{1}{2} b(c)\left(H-H_{0}(c)\right)^{2} \mathrm{~d} A .
$$

Note that this functional has also been used to model the energy of multicomponent vesicles, e.g., 34, 20, where in that case $c$ denotes the concentration of one of the lipid components of the membrane. Here, we assume for simplicity that $b$ and $H_{0}$ are linear functions of $c$. Other choices are discussed in Appendix A.1. In Appendix A.2 we derive a sharp interface model of an inextensible membrane with CIMs in viscous fluids using energy variation. 


\subsection{Diffuse interface model}

The diffuse interface method, also known as the phase field method, introduces an auxiliary field $\phi$ that distinguishes the membrane interior from the exterior. The membrane is modeled by a narrow, diffuse layer. An equation is posed for the phase field function $\phi$, which is nonlinearly coupled to the fluid equations. Near the interface, $\phi$ can be approximated by

$$
\phi(t, \mathbf{x}):=\tanh \left(\frac{-r(t, \mathbf{x})}{\sqrt{2} \epsilon}\right)
$$

where $\epsilon$ characterizes the thickness of the diffuse interface and $r(t, \mathbf{x})$ denotes the signed-distance function between $\mathbf{x} \in \Omega$ and its nearest point on $\Gamma(t)$. Taking $r$ to be negative inside the membrane, we label the inside with $\phi \approx 1$ and the outside with $\phi \approx-1$. The interface $\Gamma(t)$ is implicitly defined as the zero level set of $\phi$. Further, let $\mathbf{n}=\nabla \phi /|\nabla \phi|$ denote the membrane normal and $\mathbf{m}$ the outer normal to the computational domain. The complete setting is depicted in Fig. 2 Although Fig. 2 depicts the case of a cylindrical computational domain, we will derive the governing equations for general 3D-domains in the following.

\subsubsection{Diffuse interface energy}

A diffuse interface version of the standard Helfrich energy (1) of a clean membrane was proposed in [11, 10:

$$
\mathcal{E}=\int_{\Omega} \frac{1}{2} \frac{3}{4 \sqrt{2} \epsilon} b_{m}\left(\epsilon \Delta \phi-\frac{1}{\epsilon}\left(\phi^{2}-1\right)\left(\phi+\sqrt{2} \epsilon H_{0}\right)\right)^{2} \mathrm{~d} x,
$$

where $\Omega=\Omega_{1} \cup \Omega_{2} \cup \Gamma$ is the computational domain. The scaling factor $3 / 4 \sqrt{2} \epsilon$ is chosen to match the sharp interface energy in the thin interface limit 11.

To approximate the energy of a partially-covered membrane embedded in two fluids, we now assume that the bending stiffness and spontaneous curvature depend on $c$. We also account for inertial effects by adding the kinetic energy. The total energy is

$$
\mathcal{E}=\int_{\Omega} \frac{1}{2} \frac{3}{4 \sqrt{2} \epsilon} b(c)\left(\epsilon \Delta \phi-\frac{1}{\epsilon}\left(\phi^{2}-1\right)\left(\phi+\sqrt{2} \epsilon H_{0}(c)\right)\right)^{2}+\frac{1}{2} \rho|\mathbf{u}|^{2} \mathrm{~d} x,(5)
$$

where $\mathbf{u}$ denotes the velocity field. Next, we use an energy variational argument to derive a thermodynamically consistent system of evolution equations for the diffuse interface, the fluid flow and the CIMs.

\subsubsection{Evolution equations}

To simplify notation let us introduce the following abbreviations:

$$
\begin{aligned}
\tilde{b}(c) & =\frac{3}{4 \sqrt{2}} b(c) \\
f(\phi, c) & =\epsilon \Delta \phi-F(\phi, c) \\
F(\phi, c) & =\frac{1}{\epsilon}\left(\phi^{2}-1\right)\left(\phi+\sqrt{2} \epsilon H_{0}(c)\right)
\end{aligned}
$$


The time derivative of the energy (5) can be written as

$$
\begin{aligned}
\partial_{t} \mathcal{E} & =\int_{\Omega} \frac{1}{\epsilon} \tilde{b} f\left(\epsilon \Delta \partial_{t} \phi-\frac{\partial F}{\partial \phi} \partial_{t} \phi-\frac{\partial F}{\partial c} \partial_{t} c\right)+\frac{1}{2 \epsilon} \tilde{b}^{\prime}(c) f^{2} \partial_{t} c+\rho \partial_{t} \mathbf{u} \cdot \mathbf{u} \mathrm{d} x \\
& =\int_{\Omega}\left(\Delta(\tilde{b} f)-\frac{1}{\epsilon} \tilde{b} f \frac{\partial F}{\partial \phi}\right) \partial_{t} \phi+\left(\frac{1}{2 \epsilon} \tilde{b}^{\prime}(c) f^{2}-\frac{1}{\epsilon} \tilde{b} f \frac{\partial F}{\partial c}\right) \partial_{t} c+\rho \partial_{t} \mathbf{u} \cdot \mathbf{u} \mathrm{d} x
\end{aligned}
$$

where we used integration by parts and dropped the boundary integrals by assuming the two boundary conditions $\mathbf{m} \cdot \nabla \phi=0, \phi=\phi(t=0)$ on $\delta \Omega$.

We assume balance laws for momentum and mass conservation, although with forces that need to be determined consistently with the second law of thermodynamics (thermodynamic consistency). It is known that the lipid bilayer of a membrane resists any in-plane compression or stretching. Mathematically this leads to the constraint of constant local surface area, which is equivalent to $\nabla_{\Gamma} \cdot \mathbf{u}=\mathbf{P}: \nabla \mathbf{u}$ at the membrane where $\mathbf{P}=\mathbf{I}-\mathbf{n} \otimes \mathbf{n}$ is the surface projection operator and $\nabla_{\Gamma}=\mathbf{P} \nabla$ is the surface gradient. This is also termed surface incompressibility or inextensibility. To ensure local inextensibility at the fluid-fluid interface we use the the approach presented in [2. Accordingly the Navier-Stokes equations are enriched by an additional variable $\lambda$ which acts as a Lagrange multiplier and can also be interpreted as a surface tension. The inextensibility constraint is then added as additional equation restricted to the interface. The resulting Navier-Stokes system reads:

$$
\begin{aligned}
\rho\left(\partial_{t} \mathbf{u}+\mathbf{u} \cdot \nabla \mathbf{u}\right)-\nabla \cdot(\nu \mathbf{D})+\nabla p & =\nabla \cdot(|\nabla \phi| \mathbf{P} \lambda)+\mathbf{F}, \\
\nabla \cdot \mathbf{u} & =0 \\
\xi \epsilon^{2} \nabla \cdot\left(\phi^{2} \nabla \lambda\right)+|\nabla \phi| \mathbf{P}: \nabla \mathbf{u} & =0
\end{aligned}
$$

where $\mathbf{D}=\nabla \mathbf{u}+\nabla \mathbf{u}^{T}$ is the rate of strain tensor. The first term on the left hand side of Eq. (13) provides a harmonic extension of the tension $\lambda$ off the interface, with $\xi$ being a regularization parameter, see [2]. The force $\mathbf{F}$ is yet unspecified and will be determined below to ensure thermodynamic consistency. The sharp interface equations corresponding to Eqs. 11]- 13 are given in the Appendix in Eqs. A-12 A-14. When inertial forces are small, the Stokes equations, which are simpler, could be solved instead of the full Navier-Stokes equations. The Stokes system also has an energy functional and an analogous set of diffuse interface equations can be derived.

We assume that the CIM concentration satisfies an advection equation, which is reasonable due to the very small interfacial diffusion of large molecules such as clathrin (see previous section). This assumption will be relaxed later to incorporate molecular diffusion of CIMs on the membrane.

$$
\partial_{t} c+\mathbf{u} \cdot \nabla c=0 . \quad \text { in } \Omega
$$

Finally, for the phase field variable we assume convection together with a yet unspecified source term.

$$
\partial_{t} \phi+\mathbf{u} \cdot \nabla \phi=-\gamma g, \quad \text { in } \Omega
$$


where $\gamma$ is a positive mobility constant. By plugging in the balance laws (11), (14), 15 into the energy time derivative we obtain after integration by parts

$$
\begin{aligned}
\partial_{t} \mathcal{E}=\int_{\Omega}\left(\Delta(\tilde{b} f)-\frac{1}{\epsilon} \tilde{b} f \frac{d F}{d \phi}\right)(-\mathbf{u} \cdot \nabla \phi-\gamma g)-\mathbf{u} \cdot\left(\frac{1}{2 \epsilon} \tilde{b}^{\prime}(c) f^{2}-\frac{1}{\epsilon} \tilde{b} f \frac{d F}{d c}\right) \nabla c \\
+\mathbf{u} \cdot(-\mathbf{u} \cdot \nabla \mathbf{u}+\nabla \cdot(\nu \mathbf{D})-\nabla p+\nabla \cdot(|\nabla \phi| \mathbf{P} \lambda)+\mathbf{F}) \mathrm{d} x
\end{aligned}
$$

Further integration by parts gives

$$
\begin{aligned}
\partial_{t} \mathcal{E}=\int_{\Omega}( & \left.\Delta(\tilde{b} f)-\frac{1}{\epsilon} \tilde{b} f \frac{d F}{d \phi}\right)(-\mathbf{u} \cdot \nabla \phi-\gamma g)-\mathbf{u} \cdot\left(\frac{1}{2 \epsilon} \tilde{b}^{\prime}(c) f^{2}-\frac{1}{\epsilon} \tilde{b} f \frac{d F}{d c}\right) \nabla c \\
- & \frac{\nu}{2}|\mathbf{D}|^{2}+p \nabla \cdot \mathbf{u}-|\nabla \phi| \mathbf{P}: \nabla \mathbf{u} \lambda+\mathbf{u} \cdot \mathbf{F} \mathrm{d} x
\end{aligned}
$$

where the boundary integrals vanish by assuming the boundary conditions $\lambda=0$ and $\mathbf{m} \cdot(\nu \mathbf{D}-p \mathbf{I})=0$ or $\mathbf{u}=0$ on $\delta \Omega$. Next, inserting (12) and (13) yields

$$
\begin{aligned}
\partial_{t} \mathcal{E}=\int_{\Omega} & \left(\Delta(\tilde{b} f)-\frac{1}{\epsilon} \tilde{b} f \frac{d F}{d \phi}\right)(-\mathbf{u} \cdot \nabla \phi-\gamma g)-\mathbf{u} \cdot\left(\frac{1}{2 \epsilon} \tilde{b}^{\prime}(c) f^{2}-\frac{1}{\epsilon} \tilde{b} f \frac{d F}{d c}\right) \nabla c \\
& -\frac{\nu}{2}|\mathbf{D}|^{2}-\xi \epsilon^{2} \phi^{2}|\nabla \lambda|^{2}+\mathbf{u} \cdot \mathbf{F} \mathrm{d} x
\end{aligned}
$$

By choosing

$$
\begin{aligned}
g & =\Delta(\tilde{b} f)-\frac{1}{\epsilon} \tilde{b} f \frac{d F}{d \phi} \\
\mathbf{F} & =g \nabla \phi+\left(\frac{1}{2 \epsilon} \tilde{b}^{\prime}(c) f^{2}-\frac{1}{\epsilon} \tilde{b} f \frac{d F}{d c}\right) \nabla c
\end{aligned}
$$

we obtain a non-increasing energy:

$$
\partial_{t} \mathcal{E}=\int_{\Omega}-\gamma g^{2}-\frac{\nu}{2}|\mathbf{D}|^{2}-\xi \epsilon^{2} \phi^{2}|\nabla \lambda|^{2} \mathrm{~d} x,
$$

Hence, a thermodynamically consistent evolution of a partially-covered membrane is given by the coupled inextensible Navier-Stokes-Willmore problem in Eqs. (11)-(15) with $g$ and $\mathbf{F}$ from $(19)$ and (20), respectively. The derivation of the corresponding sharp interface equations is given in the Appendix A.2.

\subsection{The molecule advection equation}

In the previous section we assumed a pure advection for the concentration of CIMs in Eq. (14). We can extend this equation to consider a small amount of 
diffusion in the tangential direction to account for diffusional transport of CIMs along the interface:

$$
\partial_{t} c+\mathbf{u} \cdot \nabla c-D_{c} \Delta_{\Gamma} c=0
$$

We note that using this simple form of Fickian interface diffusion no longer guarantees that the energy $\mathcal{E}$ is non-increasing. While a more complicated surface flux could be chosen to ensure that $\mathcal{E}$ is non-increasing, we take the surface diffusivity $D_{c}$ to be small, which limits the effects of diffusion. Alternatively, if the membrane solely contains areas of no coverage $(c=0)$ and full coverage $(c=1)$, a Cahn-Hilliard equation can be used to maintain these distinct values with a sharp transition in between. This is particularly useful if large membrane molecules are considered (see Sec. 2p. The surface Cahn-Hilliard equation reads

$$
\begin{aligned}
\partial_{t} c+\mathbf{u} \cdot \nabla c & =D_{c} \Delta_{\Gamma} \mu \\
\mu & =-\epsilon \Delta_{\Gamma} c+\epsilon^{-1}\left(4 c^{3}-6 c^{2}+2 c\right)
\end{aligned}
$$

Note that if $\mu$ is constant, so that the CIM concentration is in local equilibrium, then this equation reduces to the advection equation. Typically, deviations from local equilibrium are small. Both the Fickian and Cahn-Hilliard forms of the CIM evolution equation enable the use of finite-element or central difference approximations without additional numerical stabilization such as the streamline diffusion method or upwind discretizations.

Straightforward discretizations of these equations may not conserve the mass of the molecules along the interface. Note, that the continuous advection equation conserves mass along the interface if inextensibility is perfectly fulfilled, but this is not necessarily carried over to the discrete case, where inextensibility and incompressibility are subject to spatial discretization errors. To deal with the problem of interfacial mass conservation, we use the diffuse interface approach from [31. In this approach, the equations are restricted to the interface by multiplying with an approximation to the interface delta function (here: taken to be $|\nabla \phi|)$. The advection-diffusion equation 22 becomes

$$
\partial_{t}(|\nabla \phi| c)+\nabla \cdot(|\nabla \phi| \mathbf{u} c)-D_{c} \nabla \cdot(|\nabla \phi| \nabla c)=0
$$

and the Cahn-Hilliard equation (23)-(24) becomes

$$
\begin{array}{r}
\partial_{t}(|\nabla \phi| c)+\nabla \cdot(|\nabla \phi| \mathbf{u} c)-D_{c} \nabla \cdot(|\nabla \phi| \nabla \mu)=0 \\
|\nabla \phi| \mu+\epsilon \nabla \cdot(|\nabla \phi| \nabla c)-\epsilon^{-1}|\nabla \phi|\left(4 c^{3}-6 c^{2}+2 c\right)=0
\end{array}
$$

If the diffusion constant $D_{c}$ is large enough, the diffuse interface approach 31] ensures that $c$ is extended off $\Gamma$ constant in normal direction. This property is very important, since the bending stiffness and spontaneous curvature strongly depend on $c$. Having variations in these quantities across the interface makes no sense physically. However, for the very small $D_{c}$ that we consider here, it cannot be guaranteed that $c$ is constant in normal direction. Therefore it is necessary to introduce additional normal diffusion that averages $c$ across the 
diffuse interface and results in constant extensions of $c$ off the interface, without affecting the accuracy of the solution on the interface. This approach has also been used in [13]. The resulting equation reads

$$
\partial_{t}(|\nabla \phi| c)+\nabla \cdot(|\nabla \phi| \mathbf{u} c)-D_{c} \nabla \cdot(|\nabla \phi| \nabla c)-D_{n} \nabla \cdot(|\nabla \phi| \mathbf{n} \otimes \mathbf{n} \cdot \nabla c)=0
$$

where $D_{n}$ is the normal diffusion constant and should be chosen larger than $D_{c}$. In practice we empirically determine $D_{n}$ by increasing its value until a constant extension of $c$ across the interface is ensured. Note, that in principle the accuracy and conservation of $c$ along the membrane are not affected by the normal diffusion, even if $D_{n}$ is very large.

The equations 28) or 26)-27) both guarantee exact conservation of molecule mass on the interface: $\partial_{t} \int|\nabla \phi| c \mathrm{~d} x=0$, in contrast to the original equations. With appropriate discretization this property is also carried over to the fully discrete system. Matched asymptotic analysis shows convergence of Eq. (28) to the original equation (22) and convergence of Eqs. (26)-(27) to Eqs. (23)-(24), see 31 .

\subsection{Improving the accuracy of the inextensibility constraint}

The strong tangential forces that arise from tangential gradients of the CIMs in $\mathbf{F}$ can lead to problems with satisfying the inextensibility condition in the discrete system. Hence, small stretching or compression of the membrane may occur and, if allowed to accumulate over time, these errors can strongly influence the simulation results. Therefore we use a relaxation mechanism to correct accumulated local errors in the inextensibility as proposed in [2]. Following this approach, we introduce an additional variable $s(x, t)$ to measure the accumulated local stretching and then we modify the RHS of Eq. (13) as described below. Initially $s(x, t)$ is set equal to 1 everywhere and for $t>0, s(x, t)$ is evolved by an advection-diffusion equation on the interface:

$$
\partial_{t} s+\mathbf{u} \cdot \nabla s+s \nabla_{\Gamma} \cdot \mathbf{u}-D_{s} \Delta_{\Gamma} s=0
$$

This equation ensures conservation of $s$ along the interface and indeed $s$ can be thought of as an interfacial species concentration. This implies that $s>1$ in regions where the interface is compressed and $s<1$ where the interface is stretched. Eq. 29 can be approximated using a diffuse interface formulation as done in the previous section for the CIMs:

$$
\partial_{t}(|\nabla \phi| s)+\nabla \cdot(|\nabla \phi| \mathbf{u} s)-D_{s} \nabla \cdot(|\nabla \phi| \nabla s)-D_{n} \nabla \cdot(|\nabla \phi| \mathbf{n} \otimes \mathbf{n} \cdot \nabla s)=0 .
$$

The idea is now to not require that inextensibility holds $\left(\nabla_{\Gamma} \cdot \mathbf{u}=\mathbf{0}\right)$ but instead $\nabla_{\Gamma} \cdot \mathbf{u}=\zeta(s-1) / s$ on $\Gamma$. Hence a slightly stretched or compressed interface will be driven back to a relaxed state. In the diffuse interface context, the relaxation model is realized by adding the term $\zeta|\nabla \phi|(s-1) / s$ to the RHS of Eq. (13).

In our simulations we will consider a part of the membrane, which implies that the membrane cuts the domain boundary. Hence, the membrane area is 
allowed to change, due to flow into the computational domain. In this case a special boundary is necessary. Conservation of $s$ on the membrane requires that the change in membrane area is equal to the amount of membrane flowing in from the boundaries. The membrane area can be expressed in terms of $s$ by $\int_{\Omega}|\nabla \phi| s d x$. Hence, $\partial_{t} \int_{\Omega}|\nabla \phi| s d x=\int_{\partial \Omega}-\mathbf{m} \cdot \mathbf{P u}|\nabla \phi| d S$, where $\mathbf{m}$ is the outer normal to the computational domain and we have assumed that the membrane outside of the computational is unstretched $(s=1)$. To ensure conservation (locally) we use

$$
|\nabla \phi| \mathbf{m} \cdot\left(\mathbf{u} s-D_{s} \nabla s-D_{n} \mathbf{n} \otimes \mathbf{n} \cdot \nabla s\right)=|\nabla \phi| \mathbf{m} \cdot \mathbf{P u} \quad \text { on } \partial \Omega .
$$

as boundary condition to Eq. 30 .

\subsection{Governing equations}

Let us now summarize the governing equations derived in the previous sections. The diffuse interface model for a membrane partially covered by CIMs consists of the following coupled system:

1. The inextensible, incompressible Navier-Stokes equations for the fluid velocity

$$
\begin{aligned}
& \rho\left(\partial_{t} \mathbf{u}+\mathbf{u} \cdot \nabla \mathbf{u}\right)-\nabla \cdot(\nu \mathbf{D})+\nabla p=\nabla \cdot(|\nabla \phi| \mathbf{P} \lambda) \\
& +g \nabla \phi+\frac{3}{4 \sqrt{2}}\left(\frac{1}{2 \epsilon} b^{\prime}(c) f^{2}-\frac{\sqrt{2}}{\epsilon^{2}} b f\left(\phi^{2}-1\right) \epsilon H_{0}^{\prime}(c)\right) \nabla c \\
& \nabla \cdot \mathbf{u}=0 \\
& \xi \epsilon^{2} \nabla \cdot\left(\phi^{2} \nabla \lambda\right)+|\nabla \phi| \mathbf{P}: \nabla \mathbf{u}=\zeta|\nabla \phi|(s-1) / s
\end{aligned}
$$

with boundary conditions $\lambda=0$ and $\mathbf{u}=0$ or $(\nu \mathbf{D}-p \mathbf{I}) \cdot \mathbf{m}=0$ on $\delta \Omega$.

2. The evolution equation for the phase field variable

$$
\begin{aligned}
\partial_{t} \phi+\mathbf{u} \cdot \nabla \phi & =-\gamma g \\
g & =\frac{3}{4 \sqrt{2}}\left(\Delta(b f)-\epsilon^{-2} b f\left(3 \phi^{2}-1+2 \sqrt{2} \phi \epsilon H_{0}(c)\right)\right) \\
f & =\epsilon \Delta \phi-\epsilon^{-1}\left(\phi^{2}-1\right)\left(\phi+\sqrt{2} \epsilon H_{0}(c)\right)
\end{aligned}
$$

with boundary condition $\mathbf{m} \cdot \nabla \phi=0$ and $\phi=\phi(t=0)$ on $\delta \Omega$.

3. The evolution equation for the stretching measure $s$ on the diffuse interface

$$
\partial_{t}(|\nabla \phi| s)+\nabla \cdot(|\nabla \phi| \mathbf{u} s)-D_{s} \nabla \cdot(|\nabla \phi| \nabla s)-D_{n} \nabla \cdot(|\nabla \phi| \mathbf{n} \otimes \mathbf{n} \cdot \nabla s)=0
$$

with boundary condition 31.

4. The evolution equation for the molecule concentration $c$ on the diffuse interface

$$
\partial_{t}(|\nabla \phi| c)+\nabla \cdot(|\nabla \phi| \mathbf{u} c)-D_{c} \nabla \cdot(|\nabla \phi| \nabla c)-D_{n} \nabla \cdot(|\nabla \phi| \mathbf{n} \otimes \mathbf{n} \cdot \nabla c)=0
$$


or if the Cahn-Hilliard equation is used,

$$
\begin{array}{r}
\partial_{t}(|\nabla \phi| c)+\nabla \cdot(|\nabla \phi| \mathbf{u} c)-D_{c} \nabla \cdot(|\nabla \phi| \nabla \mu)=0 \\
|\nabla \phi| \mu+\epsilon \nabla \cdot(|\nabla \phi| \nabla c)-\epsilon^{-1}|\nabla \phi|\left(4 c^{3}-6 c^{2}+2 c\right)=0
\end{array}
$$

with no flux boundary conditions.

\section{Numerical method}

To solve the system of equations numerically we split the time interval $I=$ $[0, T]$ into equidistant time instants $0=t_{0}<t_{1}<\ldots$ and define the time steps $\tau:=t_{n+1}-t_{n}$ (adaptive time steps could also be used). We define the discrete time derivative $\partial_{t} \cdot{ }^{n+1}:=\left(\cdot{ }^{n+1}-{ }^{n}\right) / \tau$, where the upper index denotes the time step number. We will use the notation $(\cdot)^{n}$ to denote that all timedependent variables in the brackets are evaluated at time step $n$. To correct any accumulated errors in the inextensibility, we choose the relaxation speed $\zeta=1 / \tau$ in Eq. (34) as in 2.

The numerical approach for each subproblem is adapted from existing algorithms for the Navier-Stokes equations and the Helfrich model. We solve the overall system using an operator splitting approach meaning that we solve sequentially the series of sub-problems listed below. In particular, the NavierStokes equations are implicitly coupled to the inextensibility constraint and the Willmore problem for the phase field variable while the equations for the CIM concentration $c$ and the stretching variable $s$ are solved separately.

Hence, at each time step we solve

1. A linear coupled system for the flow $\left(\mathbf{u}^{n+1}, p^{n+1}, \lambda^{n+1}\right)$ and the Willmore problem $\left(\phi^{n+1}, g^{n+1} f^{n+1}\right)$ :

$$
\begin{aligned}
& \rho^{n}\left(\partial_{t} \mathbf{u}^{n+1}+\mathbf{u}^{n} \cdot \nabla \mathbf{u}^{n+1}\right)+\nabla p^{n+1}-\nabla \cdot\left(\nu^{n} \mathbf{D}^{n+1}\right)-\nabla \cdot\left(\left|\nabla \phi^{n}\right| \mathbf{P}^{n} \lambda^{n+1}\right) \\
& \quad=-g^{n+1} \nabla \phi^{n}+\frac{3}{4 \sqrt{2}}\left(\frac{1}{2 \epsilon} b^{\prime}(c) f^{2}-\frac{\sqrt{2}}{\epsilon^{2}} b f\left(\phi^{2}-1\right) \epsilon H_{0}^{\prime}(c)\right)^{n} \nabla c^{n}, \\
& \nabla \cdot \mathbf{u}^{n+1}=0, \\
& \xi \epsilon^{2} \nabla \cdot\left(\left(\phi^{n}\right)^{2} \nabla \lambda^{n+1}\right)+\left|\nabla \phi^{n}\right| \mathbf{P}^{n}: \nabla \mathbf{u}^{n+1}=\tau^{-1}\left|\nabla \phi^{n}\right| \frac{s^{n}-1}{s^{n}}, \\
& \partial_{t} \phi^{n+1}+\mathbf{u}^{n+1} \cdot \nabla \phi^{n}=-\gamma g^{n+1}, \\
& g^{n+1}=\frac{3}{4 \sqrt{2}}\left(\Delta\left(b^{n} f^{n+1}\right)-\epsilon^{-2} b^{n} f^{n+1}\left(3\left(\phi^{n+1}\right)^{2}-1+2 \sqrt{2} \phi^{n+1} \epsilon H_{0}\left(c^{n}\right)\right)\right) \\
& f^{n+1}=\epsilon \Delta \phi^{n+1}-\epsilon^{-1}\left(\left(\phi^{n+1}\right)^{2}-1\right)\left(\phi^{n+1}+\sqrt{2} \epsilon H_{0}\left(c^{n}\right)\right)
\end{aligned}
$$

where $\rho^{n}=\rho\left(\phi^{n}\right)=\rho_{1}\left(1+\phi^{n}\right) / 2+\rho_{2}\left(1-\phi^{n}\right) / 2$, and $\nu^{n}$ is defined analogously. Further, $\mathbf{P}^{n}=\mathbf{I}-\nabla \phi^{n} \otimes \nabla \phi^{n} /\left|\nabla \phi^{n}\right|^{2}$. In addition, we linearize the nonlinear terms using a Taylor series expansion of order one, e.g. $\left(\left(\phi^{n+1}\right)^{2}-1\right) \phi^{n+1}=\left(\left(\phi^{n}\right)^{2}-1\right) \phi^{n}+\left(3\left(\phi^{n}\right)^{2}-1\right)\left(\phi^{n+1}-\phi^{n}\right)$. 
2. The advection-diffusion equation for the stretching variable $s^{n+1}$ :

$$
\begin{gathered}
\frac{\left|\nabla \phi^{n+1}\right| s^{n+1}-\left|\nabla \phi^{n}\right| s^{n}}{\tau}+\nabla \cdot\left(\left|\nabla \phi^{n+1}\right| \mathbf{u}^{n+1} s^{n+1}\right)-D_{s} \nabla \cdot\left(\left|\nabla \phi^{n+1}\right| \nabla s^{n+1}\right) \\
-D_{n} \nabla \cdot\left(\left|\nabla \phi^{n+1}\right| \mathbf{n}^{n+1} \mathbf{n}^{n+1} \cdot \nabla s^{n+1}\right)=0
\end{gathered}
$$

with boundary condition (31).

3. The advection-diffusion equation for the species concentration $c^{n+1}$ :

$$
\begin{gathered}
\frac{\left|\nabla \phi^{n+1}\right| c^{n+1}-\left|\nabla \phi^{n}\right| c^{n}}{\tau}+\nabla \cdot\left(\left|\nabla \phi^{n+1}\right| \mathbf{u}^{n+1} c^{n+1}\right)-D_{c} \nabla \cdot\left(\left|\nabla \phi^{n+1}\right| \nabla c^{n+1}\right) \\
-D_{n} \nabla \cdot\left(\left|\nabla \phi^{n+1}\right| \mathbf{n}^{n+1} \mathbf{n}^{n+1} \cdot \nabla c^{n+1}\right)=0
\end{gathered}
$$

or if the Cahn-Hilliard equation is used:

$$
\begin{array}{r}
\frac{\left|\nabla \phi^{n+1}\right| c^{n+1}-\left|\nabla \phi^{n}\right| c^{n}}{\tau}+\nabla \cdot\left(\left|\nabla \phi^{n+1}\right| \mathbf{u}^{n+1} c^{n+1}\right)-D_{c} \nabla \cdot\left(\left|\nabla \phi^{n+1}\right| \nabla c^{n+1}\right)=0 \\
\left|\nabla \phi^{n+1}\right| \mu^{n+1}+\epsilon \nabla \cdot\left(\left|\nabla \phi^{n+1}\right| \nabla c^{n+1}\right)-\epsilon^{-1}\left|\nabla \phi^{n+1}\right|\left(4 c^{3}-6 c^{2}+2 c\right)^{n+1}=0
\end{array}
$$

Note, that the implicit coupling of Navier-Stokes and Willmore-problem increases the stability and allows larger time steps. This is in accordance with similar implicit coupling strategies for the Navier-Stokes (and other fluid flow equations) and Cahn-Hilliard equations, e.g., 43, 6, 1, 5, 9, 9 .

We solve the 3D-axisymmetric form of the governing equations using the adaptive finite element toolbox AMDiS [39] for spatial discretizations. We use $P^{2} / P^{1}$ Taylor-Hood elements for velocity and pressure, extended by a $P^{2}$ element for $\lambda$. For $\phi, f, g, s$ and $c, P^{2}$ elements are also used. The resulting linear systems of equations are solved with UMFPACK [8]. The adaptive mesh refinement and coarsening are determined by gradients in the phase field function, which ensures high numerical resolution near the diffuse interface when small values of the interface thickness $\epsilon$ are used; coarse meshes are used away from interfacial regions. We find this refinement is also sufficient to accurately capture gradients in the velocity field.

\section{Numerical results}

We use our diffuse interface model and numerical method for an initial exploration of ultrafast CIM-mediated endocytosis. We neglect several phenomena, such as the process by which CIM attach to the membrane. Here we are primarily interested in testing the efficacy of the method and exploring characteristic features, such as timescales, and dependence on parameters such as the size of the CIM-covered patch. The bending stiffness of clathrin was measured in [22] to be $b_{c}=12 b_{m}$, where $b_{m}=1 \times 10^{-19} \mathrm{~kg} \mathrm{~m}^{2} / \mathrm{s}^{2}$ is the bending stiffness of a typical uncovered membrane. For the spontaneous curvature we assume $H_{0}=(14.1 n m)^{-1}$. According to these parameters we 
choose the concentration-dependent parameters $H_{0}(c)=c \cdot(14.1 \mathrm{~nm})^{-1}$ and $b(c)=(1+11 c) \cdot 1 \times 10^{-19} \mathrm{~kg} \mathrm{m^{2 }} / \mathrm{s}^{2}$.

We assume that the initial membrane is nearly flat, axisymmetric and covered with a circular region of CIMs, as illustrated in Fig. 2, We thus implement the equations using cylindrical coordinates with the computational domain being $\Omega=[0,80] \times[0,160] \mathrm{nm}^{2}$. The circular membrane has a radius of $80 \mathrm{~nm}$. For the circular CIM region we use different radii ranging from $r=20 \mathrm{~nm}$ to $r=60 \mathrm{~nm}$. For the boundary conditions we pin the membrane at the outer domain boundary by imposing the condition $\phi^{n}=\phi(t=0)$ in the equation for $g$. Furthermore we enforce a 90 degree angle between the membrane and the domain boundary: $\mathbf{m} \cdot \nabla \phi=0$, where $\mathbf{m}$ is the normal on the domain boundary. For the velocity we apply a free stress condition almost everywhere. The only exception to this condition is made where the outer domain boundary meets the membrane. There, in a small neighborhood of the membrane we impose zero force in horizontal direction $(1,0) \cdot(\nu \mathbf{D}-p \mathbf{I}) \cdot \mathbf{m}=0$ and zero vertical velocity $\mathbf{u} \cdot(0,1)^{T}=0$. This corresponds to the pinning of the membrane, since it prevents the membrane from moving vertically, while still allowing inflow of membrane area into the computational domain. For the pressure we impose $p=0$ in one grid point, to regularize the Navier-Stokes system.

The physical and numerical parameters are given in Tab. 1. Here, we briefly comment on the choice of some of the parameters. We choose a small mobility $\gamma=6.4 \mathrm{~nm}^{3} / \mathrm{s}$ such that the phase field is predominantly advected by the flow. The value of $\epsilon=1.2 \mathrm{~nm}$ gives an interface thickness comparable to that of a real lipid interface. To improve efficiency, we use adaptive time stepping and adaptive spatial mesh refinement. Initially, we use $\tau=0.01 \mathrm{~ns}$ and double this value every 30 time steps up to $\tau_{\max }=2 \mathrm{~ns}$. An adaptive spatial grid is used to obtain high resolution near the membrane. The grid size $h$ measures the diameter of the triangles used at the interface while the grid size away from the interface is $8 h$. We take $h=1.25 \mathrm{~nm}$, which ensures that approximately five vertices on the mesh can be found across the interface.

\subsection{Sensitivity of the numerical results to parameter variations}

We first validate the numerical method by investigating the sensitivity of the results on the parameters $\epsilon, \gamma, h$ and $\tau$. The strategy is to vary one of the parameters while keeping the others fixed at the values given in Tab. 1 . However, when $\epsilon$ is varied the grid size is simultaneously adapted to ensure that the interface is resolved well enough by the grid. Also when the grid size is decreased a smaller time step size is used: $\tau=0.1 \mathrm{~ns}$. This was found to be necessary for solvability of the equations if the grid is refined and also ensures that the error in time stepping is small.

Simulations are conducted with the Cahn-Hilliard model for the molecule concentration equation. In all the simulations the membrane develops an outof-plane deformation, forms a bud and finally evolves to stationary configuration consisting of a vesicle connected to the membrane by a thin neck. Since the stationary states for all parameters are quite similar, we measure the dependency of the parameters in the transient state at the early time $t=0.5 \mu \mathrm{s}$ when the 


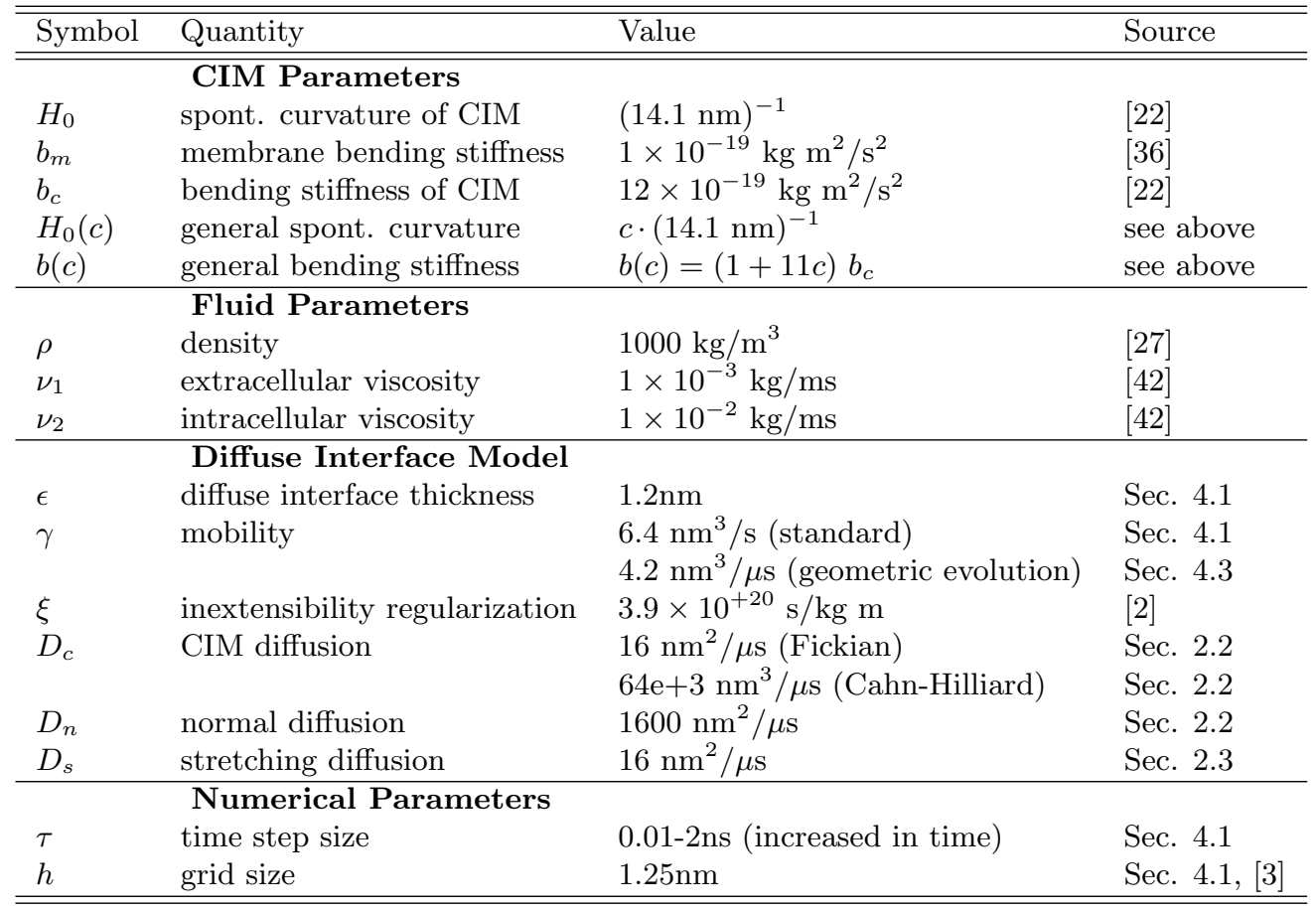

Table 1: Physical and numerical parameters used in the simulations. 


\begin{tabular}{rr|rr|rr|rr}
\hline$\gamma$ & rel. change & $\epsilon$ & rel. change & $\tau$ & rel. change & $\mathrm{h}$ & rel. change \\
\hline $12.8 \mathrm{~nm}^{3} / \mathrm{s}$ & $1.84 \%$ & $2.40 \mathrm{~nm}$ & $8.53 \%$ & $2.00 \mathrm{~ns}$ & $0.74 \%$ & $1.77 \mathrm{~nm}$ & $3.74 \%$ \\
$6.4 \mathrm{~nm}^{3} / \mathrm{s}$ & $0.44 \%$ & $1.70 \mathrm{~nm}$ & $3.67 \%$ & $1.00 \mathrm{~ns}$ & $0.47 \%$ & $1.25 \mathrm{~nm}$ & $2.14 \%$ \\
$3.2 \mathrm{~nm}^{3} / \mathrm{s}$ & $0.26 \%$ & $1.20 \mathrm{~nm}$ & - & $0.50 \mathrm{~ns}$ & $0.33 \%$ & $0.88 \mathrm{~nm}$ & $0.01 \%$ \\
$1.6 \mathrm{~nm}^{3} / \mathrm{s}$ & - & & & $0.25 \mathrm{~ns}$ & - & $0.63 \mathrm{~nm}$ & - \\
\hline
\end{tabular}

Table 2: Sensitivity of the membrane area ( rel. change $=\left|A-A_{\text {ref }}\right| / A_{\text {ref }}$ ) at $t=0.5 \mu s$ to various parameters. See text for details.

membrane starts to deform. The speed with which this occurs is highly dependent on the parameters. The out-of-plane deformation is accompanied with an increase of membrane area over time, which makes the membrane area to be an excellent quantity to measure. Since we do not have an exact solution (convergence of the inextensible part of the membrane dynamics algorithm was shown previously in 2]), we measure the relative change between the area $A$ for a particular parameter value and a reference value $A_{\text {ref }}$, which corresponds to the membrane area from the smallest value of the respective parameter: rel. change $=\left|A-A_{\text {ref }}\right| / A_{\text {ref. }}$. The rate of decrease of this quantity gives an indication of the rate of convergence of the algorithm.

Tab. 2 shows the sensitivity of the membrane area to different model parameters $(\gamma, \epsilon)$ and numerical parameters $(h, \tau)$, respectively. The relative changes in all of the parameters seems to converge to zero for decreasing parameter values with rates that vary from sub-to-super linear. To better judge the significance of the given relative values we note that the average interface area at $t=0.5 \mu \mathrm{s}$ is approximately $A=2.27 \times 10^{4} \mathrm{~nm}^{2}$. The membrane area is most sensitive to the interface thickness and grid size parameters $\epsilon$ and $h$, whereas the relative changes in area due to time step $\tau$ and mobility $\gamma$ are comparatively small.

\subsection{Molecule diffusion vs. Cahn-Hilliard surface dynamics}

Next we investigate the differences between the two models of molecule transport. For classical surface Fickian diffusion we choose the diffusion constants $D_{c}=16 \mathrm{~nm}^{2} / \mu s$ and $D_{n}=1600 \mathrm{~nm}^{2} / \mu s$ in Eq. (39). Having $D_{n}$ one hundred times larger than $D_{c}$ ensures that $c$ is approximately constant in normal direction away from the interface. The value of $D_{c}$ is chosen as small as possible, e.g., a smaller value of $D_{c}$ would require additional advection stabilization. The Cahn-Hilliard model allows a larger diffusion since the Cahn-Hilliard equation maintains sharp gradients in the molecule concentration. Accordingly, we use $D_{c}=64 \times 10^{+3} \mathrm{~nm}^{3} / \mu \mathrm{s}$ in Eq. 40

Fig. 3 shows the time evolution of the membranes colored by the molecule concentration using the Cahn-Hilliard model (top) and the Fickian model (bottom). Due to the presence of the CIM and its influence on the bending stiffness and preferred curvature, the membrane is initially very tense. To relieve this tension the membrane develops an out-of-plane deformation localized at the 


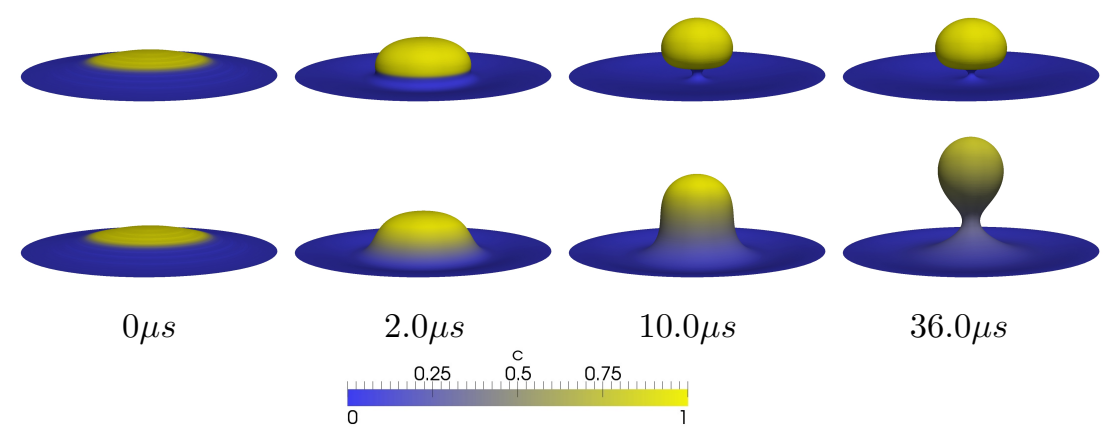

Figure 3: Comparison of membrane evolution and CIM concentrations $c$ when the curvatureinducing molecules are transported by the surface Cahn-Hilliard model (top) or Fickian surface diffusion (bottom). The Cahn-Hilliard model maintains sharp gradients of CIMs between the distinct values of 1 (covered) and 0 (uncovered) and produces a steady-state configuration consisting of a compact vesicle connected to the membrane by a small neck. Fickian diffusion decreases the gradients of the CIMs that transiently produces longer necks and more deformed vesicles.

region covered with CIMs. The bud grows to form a vesicle, which remains connected to the rest of the membrane by a neck.

When the Cahn-Hilliard model is used (top), steep gradients in the CIM concentration are maintained in time. On the other hand, when the Fickian model is used (bottom), the gradients in the CIM concentration decrease due to dissipation. Consequently, in the Cahn-Hilliard case, the dynamics are faster, the resulting neck is smaller and the vesicle is more compact compared to that obtained using Fickian model. In addition, the Cahn-Hilliard evolution leads to a non-trivial stationary state of the system. However, the membrane and CIM concentration continue to evolve in the Fickian model up to the final time $t=36.0 \mu \mathrm{s}$. Moreover, in the Fickian case, the only steady state consists of a uniformly distributed CIM concentration on a flat membrane. In the following we only focus on the Cahn-Hilliard equation for the CIMs since this model is appropriate for large, slowly diffusing molecules such as clathrin and yields non-trivial steady-states and distinct endocytosing vesicles.

\subsection{The influence of hydrodynamics}

At nanometer length scales the question arises whether hydrodynamics actually has an effect on the dynamics of the system. In principle one could also use a pure geometric evolution of the membrane without considering the surrounding fluids. To the best of our knowledge there is no geometric diffuse interface evolution available that includes membrane inextensibility. Hence, we drop the inextensibility constraint and evolve the membrane using Willmore-flow coupled to the diffuse interface Cahn-Hilliard equation for the molecule concentration (40)-(41). This transports the molecules and ensures their conservation along 


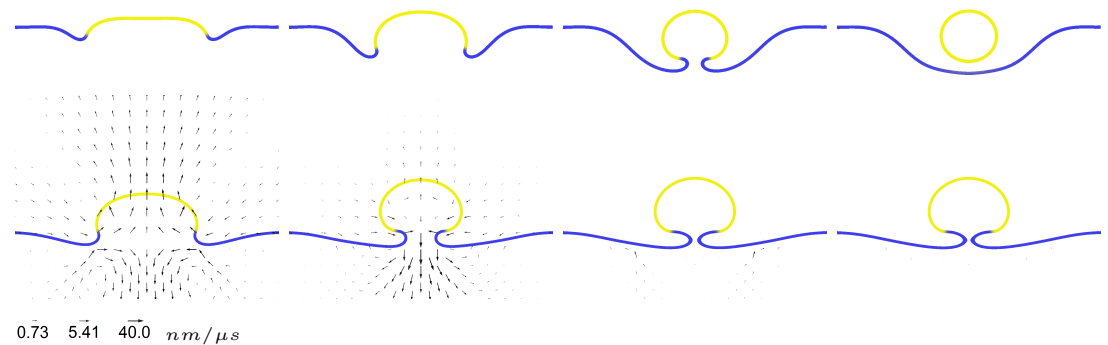

Figure 4: Comparison of the membrane evolution and CIM concentrations with (bottom) and without flow (top) at times $t=2.90 \mu \mathrm{s}, 7.46 \mu \mathrm{s}, 14.02 \mu \mathrm{s}, 16.98 \mu \mathrm{s}$ from left to right. After forming a neck the membrane pinches off without flow. This is not seen when flow is involved and the neck radius stops decreasing at a certain point. The arrows indicate the velocity direction and magnitude on a logarithmic scale.

the membrane even without flow. Accordingly, we solve only Eqs. (35)-(37) and (40)-(41) with $\mathbf{u}=0$. The parameters are as in the previous section except for the Willmore mobility, which is now the only driving force of the interface evolution. In this case, we set mobility $\gamma=4.2 \mathrm{~nm}^{3} / \mu \mathrm{s}$ so as to approximately synchronize the dynamics of the geometric and fluid models.

Fig. 4 shows a comparison of the interface evolution with (bottom) and without (top) flow. The simulation without flow shows a downwards movement of the membrane at early times since it costs no energy to push the fluid down. If flow is present this downwards movement is largely suppressed as an effect of the fluid incompressibility. After forming a neck the membrane quickly pinches off in the case of without flow. When flow is involved, this effect is not seen and the membrane rather assumes a stationary state with a stable neck. We assume inextensibility to be the main reason for this. In order for the neck radius to decrease, fluid needs to be drained out of the near contact region. Once the neck is sufficiently narrow, additional drainage would require tangential flow along the membrane due to viscosity. However this would stretch the membrane. As a result, membrane inextensibility inhibits fluid drainage in the near contact region, as can clearly be seen from the velocity vectors shown in the figure. This stabilizes the neck. This result is also in agreement with simulations presented in 2] where it was shown that inextensibility can prevent the close approach of membranes.

It is interesting that experimental results indicate that pinch-off can not be triggered by clathrin alone, but rather requires the action of another molecule called dynamin [7. In principle our model can be extended to account for the presence of dynamin and as presented in 44 an adhesive potential can be used to model fusion and fission in diffuse interface models. This will be considered in future work. 


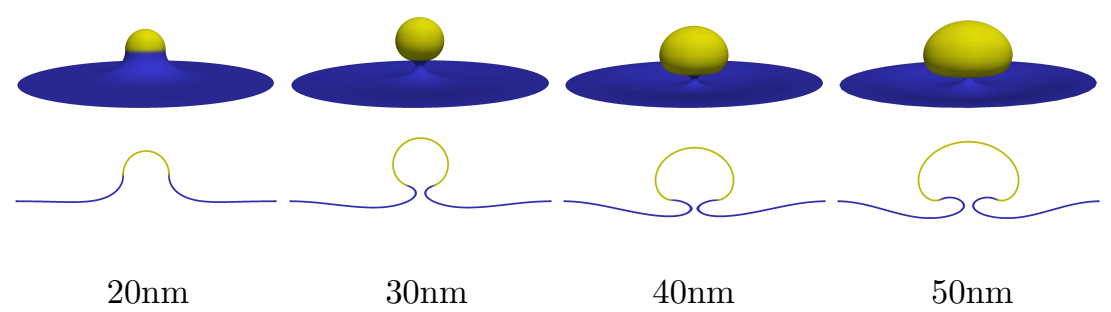

Figure 5: Stationary shapes of the membrane and CIM concentrations for different sizes of the initial CIM region using the surface Cahn-Hilliard model.

\subsection{Varying the CIM region size}

Now we investigate what effect the size of the covered region has on the dynamics and stationary state of the membrane. The radius of the initially circular CIM region is varied from $20 \mathrm{~nm}$ to $60 \mathrm{~nm}$. Fig. 5 shows the stationary shapes at $t=20.0 \mu \mathrm{s}$. We find that for small amounts of CIM (radius $\leq 20 \mathrm{~nm}$ ) the membrane deforms out-of-plane, but does not form a neck. For larger amounts (radius $28-40 \mathrm{~nm}$ ) a neck forms and the endocytosing vesicle is small and nearly spherical. If the CIM region is increased further (radius $>40 \mathrm{~nm}$ ) the vesicle assumes a mushroom-like shape.

The lateral diameter and neck radius of the stationary vesicle are displayed as a function of CIM radius in Fig. 6. Both quantities are measured at $t=20 \mu \mathrm{s}$ when the membrane has reached an (almost) stationary shape. The vesicle diameter is a linear function of the initial CIM radius as one might expect. For the neck radius the dependency on the CIM radius seems less regular. We find a critical CIM radius $r=40 \mathrm{~nm}$ which gives the most narrow neck. This value is right at the transition from spherical to mushroom-like vesicles. The occurrence of this minimal neck radius has been confirmed with refinement studies in $\epsilon$ and $h$. One possible explanation for the increasing neck radius for $r>40 \mathrm{~nm}$ is as follows: The mushroom-like shape of the vesicle more strongly inhibits fluid drainage from the neck region because more regions of the membrane are brought in close approach near the neck. As described above, this inhibits tangential fluid flow both in the neck and in nearby regions because of membrane inextensibility.

To quantify the influence of the CIM region size on the dynamics we measure the time until budding occurs. We define this to be the time at which the neck radius falls below $5 \mathrm{~nm}$. The results indicate a critical vesicle diameter of approximately $d=42 \mathrm{~nm}$ at which budding occurs fastest. This corresponds to the initial radius of the CIM region of $36 \mathrm{~nm}$. Interestingly, these results are consistent with the sizes of vesicles produced by ultrafast endocytosis in 41 . where synaptic vesicles were observed to have diameters $41.1 \pm 0.1 \mathrm{~nm}$. 

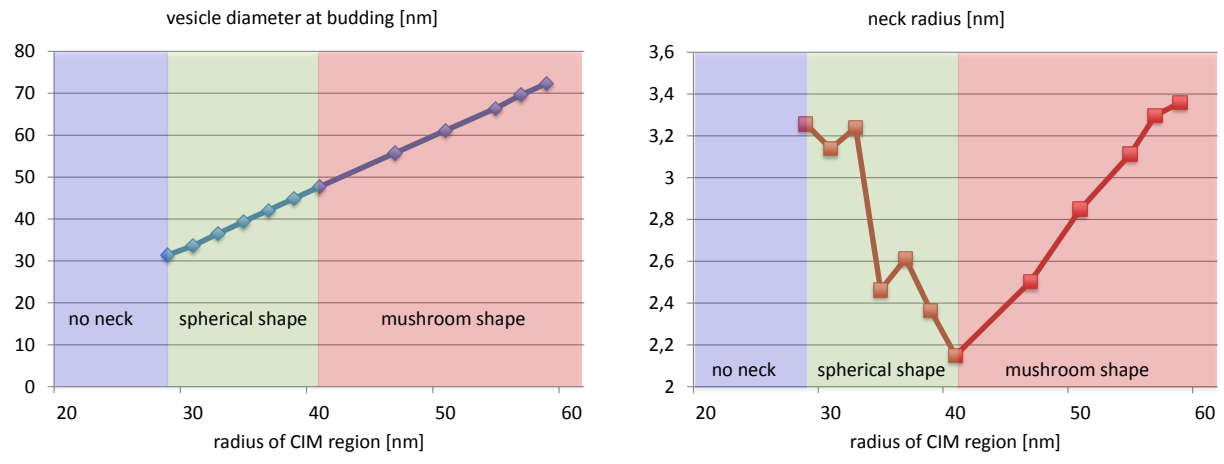

Figure 6: Diameter of the vesicle and neck radius of the stationary membrane for different sizes of the initial CIM region.

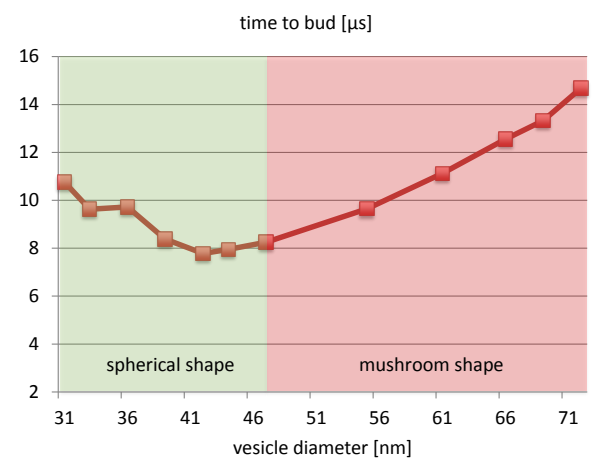

Figure 7: Time required for a bud to form for different initial radii of the CIM region. 


\section{Conclusions}

Using an energy variation methodology, we have developed new diffuse interface models for the dynamics of inextensible vesicles in a viscous fluid with stiff, curvature-inducing molecules that influence the bending stiffness and spontaneous curvature of the membrane. The model couples the Navier-Stokes equations with membrane-induced bending forces with equations for molecule transport and for a Lagrange multiplier to enforce local inextensibility. Following [2], the Lagrange multiplier is harmonically extended off the interface and a relaxation scheme is used that dynamically corrects local stretching/compression errors thereby preventing their accumulation. This is critical to accurately capturing hydrodynamic effects during endocytosis.

Two forms of surface transport equations were considered: Fickian surface diffusion and Cahn-Hilliard surface dynamics. The surface equations were reformulated using the diffuse interface method where diffusion in the normal direction is added to ensure that the CIM concentration is extended off the interface constant in the normal direction, which improves accuracy and stability of the method.

Using an adaptive finite element method, the system was solved in 3D axisymmetric geometries with boundary conditions that enabled the transport of membrane into and out of the computational domain. The membrane in the computational domain is thus interpreted as only a part of the cell membrane, which is much larger (e.g., micron scale).

The results showed that hydrodynamics can indeed enable the rapid formation of a small vesicle attached to the membrane by a narrow neck. When the Fickian model is used, this is a transient state with the steady state being a flat membrane with a uniformly distributed molecule concentration due to diffusion. When the Cahn-Hilliard model is used, molecule concentration gradients are sustained, the neck stabilizes due to membrane inextensibility and the system evolved to a steady-state with a small, compact vesicle attached to the membrane.

The steady-state vesicle morphology in the Cahn-Hilliard model was found to depend on the initial coverage of CIMs on the membrane. When the coverage is too small, no neck formed. In an intermediate range of CIM coverages, the vesicle acquired a spherical shape. When the CIM coverage was large, the vesicle attained a mushroom-like shape as regions of negative curvature developed. By varying the membrane coverage of molecules in the Cahn-Hilliard model, we found that there is a critical (smallest) neck radius and a critical (fastest) budding time. We found that the vesicle diameter corresponding to the fastest budding was consistent with the typical sizes of synaptic vesicles produced by ultrafast endocytosis in experiments 41 .

An important, and potentially rate limiting step in CIM-orchestrated endocytosis and membrane fission is the transport of CIMs in the bulk fluid to and from the membrane as well as the details of adsorption and desorption processes at the membrane. While we have not considered these effects here, they can straightforwardly be incorporated in our formulation using the diffuse interface 
methodology developed in [37, 38 to couple bulk and surface transport. A similar methodology can also be used to investigate the influence of the budding dynamics, vesicle morphologies and corresponding fluid flows on the transport of soluble molecules and cargo from the exterior to the interior of a cell. Another important feature that should be investigated is the excess energy, or line tension, that may be associated with a CIM/clean interface on the membrane. Further, since the spatial scales are small, thermal fluctuations may also play a role in CIM transport and the membrane dynamics. Fully three dimensional geometries should also be considered as small non-axisymmetric perturbations could influence the results. These and other processes will be considered in future work.

\section{Acknowledgments}

S.A. acknowledges support of the German Science Foundation within SPP 1506 Al1705/1 and by the European Commission within FP7-PEOPLE-2009IRSES PHASEFIELD, which J.L. also ackowledges. Further, J.L. is grateful for support from the National Science Foundation Division of Mathematical Sciences and from the National Institutes of Health through grant P50GM76516 for a Center of Excellence in Systems Biology at the University of California, Irvine. Simulations were carried out at ZIH at TU Dresden and JSC at FZ Jülich. S.A. also thanks the hospitality of the Department of Mathematics at the University of California, Irvine where some of this research was conducted.

\section{Appendix}

\section{A.1. Energy of a partially-covered membrane}

In [29] the energy of a membrane with CIMs was modeled using

$$
E=\int_{\Gamma} \frac{1}{2} b_{c} c\left(H-H_{0}\right)^{2}+\frac{1}{2} b_{m} H^{2} \mathrm{~d} A,
$$

where $c$ was interpreted as a coarse-grained concentration. This is questionable for large molecules such as clathrin since the size of a clathrin molecule can be about the same order of magnitude as the considered domain size. There is also another reason why the above energy $\mathrm{A}-1$ is not necessarily a good choice. The transformation of (1) to (A-1) uses the simple ansatz $\int_{\Gamma_{c}} \cdot \mathrm{d} A=\int_{\Gamma} c \cdot \mathrm{d} A$, which is not appropriate if $c$ is a coarse-grained concentration. In fact the whole energy (A-1) must be coarse-grained to account for different bending of covered and non-covered parts of the membrane.

Based on this principle we derive an alternative form, which is a the natural coarse-grained version of the energy (1). Let us consider a small control area on the membrane $A \subset \Gamma$. Let us assume the area is small enough such that the curvature can be assumed constant along $A \cap \Gamma$ and $A \cap \Gamma_{c}$, respectively. Hence,

$$
H= \begin{cases}H_{m} & \text { in } A \cap\left(\Gamma \backslash \Gamma_{c}\right) \\ H_{c} & \text { in } A \cap \Gamma_{c}\end{cases}
$$




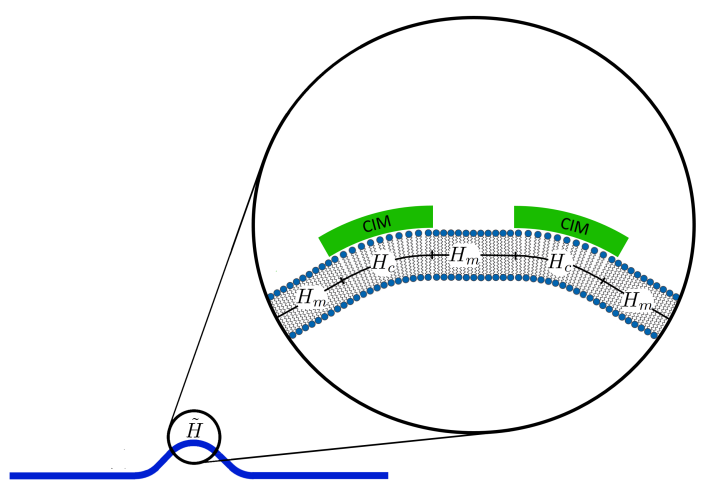

Figure A.1: Schematic of a partially covered membrane. While the observed membrane curvature is $\tilde{H}$, a close-up shows that the membrane is curved differently in covered and uncovered regions (curvature $H_{c}$ and $H_{m}$, resp.). The different curvatures are related by Eq. A-2.

Figure A.1 illustrates this setting. Since the control volume is very small, these small scale differences in the curvature will not be seen by the observer. The observed curvature is also a coarse-grained version of $H$ and can be written as a linear combination of $H_{c}$ and $H_{m}$ :

$$
\tilde{H}=H_{c}\left|A \cap \Gamma_{c}\right| /|A|+H_{m}\left|A \cap\left(\Gamma \backslash \Gamma_{c}\right)\right| /|A|
$$

Now, we can introduce the concentration $c$ of the CIM species as

$$
c=\left|A \cap \Gamma_{c}\right| /|A|,
$$

which gives

$$
\tilde{H}=c H_{c}+(1-c) H_{m}
$$

Next, consider the energy of the control volume. From Eq. (1) we obtain

$$
E_{A}=\frac{1}{2} b_{c}\left(H_{c}-H_{0}\right)^{2}|A| c+\frac{1}{2} b_{m} H_{m}^{2}|A|(1-c)+\frac{1}{2} b_{m} H_{c}^{2}|A| c .
$$

Assume that this energy is minimal, since A is small enough so that the system is in local equilibrium. Subject to Eq. $\mathrm{A}-3$ and keeping $\tilde{H}$ fixed, $E_{A}$ is minimized for

$$
H_{m}=\frac{\left(b_{c}+b_{m}\right)}{\left(b_{c}+b_{m}\right)-c \cdot b_{c}}\left(\tilde{H}-H_{0} \frac{b_{c}}{b_{c}+b_{m}}\right), \quad H_{c}=\left(\tilde{H}-H_{m} \cdot(1-c)\right) / c
$$



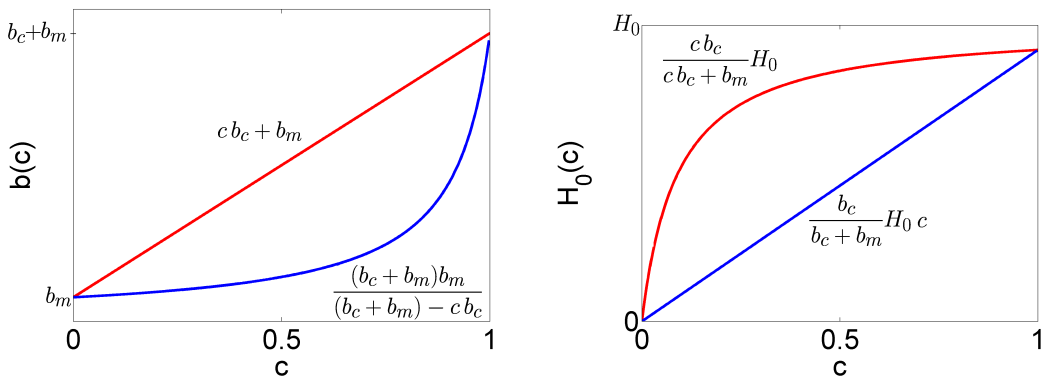

Figure A.2: A comparison of linear and nonlinear constitutive laws for the bending stiffness (left) and spontaneous curvature (right) as labelled.

which gives,

$$
E_{A}=\frac{1}{2} \frac{\left(b_{c}+b_{m}\right) b_{m}}{\left(b_{c}+b_{m}\right)-c \cdot b_{c}}\left(\tilde{H}-H_{0} \frac{b_{c}}{b_{c}+b_{m}} c\right)^{2}|A|+\mathcal{P}(c)|A|,
$$

where $\mathcal{P}(c)$ is a rational function of c, independent of $\tilde{H}$. Since the above holds for arbitrary $A$ we may conclude the energy of the whole membrane is

$$
E=\int_{\Gamma} \frac{1}{2} \frac{\left(b_{c}+b_{m}\right) b_{m}}{\left(b_{c}+b_{m}\right)-c \cdot b_{c}}\left(\tilde{H}-H_{0} \frac{b_{c}}{b_{c}+b_{m}} c\right)^{2}+\mathcal{P}(c) \mathrm{d} A
$$

In the case of an inextensible membrane $\left(\nabla_{\Gamma} \cdot \mathbf{u}=0\right)$ with purely advected CIM $\left(\partial_{t} c+\mathbf{u} \cdot \nabla c=0\right)$, the functional $\int_{\Gamma} \mathcal{P}(c) \mathrm{d} A$ will give a constant contribution to the energy, which allows one to drop this term in an energy variation argument. Hence, the spontaneous curvature of a partially-covered membrane depends linearly on $c$, while the bending stiffness is not a simple linear function of $c$. This is in contrast to the energy given in $\mathrm{A}-1$, which can be lumped to

$$
E=\int_{\Gamma} \frac{1}{2}\left(c b_{c}+b_{m}\right)\left(\tilde{H}-H_{0} \frac{c b_{c}}{c b_{c}+b_{m}}\right)^{2}+\mathcal{P}_{2}(c) \mathrm{d} A
$$

A comparison of the linear and nonlinear bending stiffnesses and spontaneous curvatures is shown in Fig. A.1. Here, we do not explore these differences in constitutive laws for the bending stiffness and spontaneous curvatures. This will be investigated in a future work.

\section{A.2. Sharp interface model}

In this section, we derive a sharp interface model for the membrane-CIM system using an energy variation argument. The approach is very similar to the diffuse interface case considered in Sec. 2.1.2. Consider the general energy of a 
partially-covered membrane together with the kinetic energy of the surrounding fluids

$$
E=\int_{\Gamma} \frac{b(c)}{2}\left(H-H_{0}(c)\right)^{2} \mathrm{~d} A+\int_{\Omega_{1} \cup \Omega_{2}} \frac{\rho}{2}|\mathbf{u}|^{2} \mathrm{~d} x .
$$

We vary the energy in $H$ and $c$ simultaneously by taking the time derivative of $E$. Using the Leibnitz rule and $\mathbf{n} \cdot \nabla c=0$, we obtain:

$$
\begin{gathered}
\partial_{t} E=\int_{\Gamma} b(c)\left(H-H_{0}(c)\right)\left(\partial_{t} H+\mathbf{n} \cdot \nabla H \mathbf{u} \cdot \mathbf{n}\right)+\frac{b(c)}{2}\left(H-H_{0}(c)\right)^{2} H \mathbf{u} \cdot \mathbf{n} \\
+\frac{\partial E}{\partial c} \partial_{t} c \mathrm{~d} A+\int_{\Omega_{1} \cup \Omega_{2}} \rho \partial_{t} \mathbf{u} \cdot \mathbf{u} \mathrm{d} x,
\end{gathered}
$$

where

$$
\frac{\partial E}{\partial c}=\frac{1}{2} b^{\prime}(c)\left(H-H_{0}(c)\right)^{2}-b(c)\left(H-H_{0}(c)\right) H_{0}^{\prime}(c) .
$$

The above expression involves the time derivative of curvature. For this quantity to make sense, $\mathrm{H}$ must be extended off $\Gamma$, at least in a small neighborhood. The natural extension is such that $H(\mathbf{x})$ for $x \notin \Gamma$ is the curvature of the unique surface containing $\mathbf{x}$ which assumes a fixed distance to $\Gamma$. Next, similar to the balance laws used in Eqs. 111)-(14), let us assume the following balance laws for momentum and mass conservation:

$$
\begin{aligned}
& \rho\left(\partial_{t} \mathbf{u}+\mathbf{u} \cdot \nabla \mathbf{u}\right)-\nabla \cdot(\nu \mathbf{D})+\nabla p=0 \quad \text { in } \Omega_{1} \cup \Omega_{2} \quad \text { (Navier-Stokes) } \\
& \nabla \cdot \mathbf{u}=0 \quad \text { in } \Omega_{1} \cup \Omega_{2} \quad \text { (incompressibility) } \\
& \mathbf{P}: \nabla \mathbf{u}=\nabla_{\Gamma} \cdot \mathbf{u}=0 \quad \text { on } \Gamma \quad \text { (inextensibility) } \\
& \partial_{t} c+\mathbf{u} \cdot \nabla c=0 \quad \text { on } \Gamma \quad \text { (species advection) }
\end{aligned}
$$

where $\mathbf{D}=\nabla \mathbf{u}+\nabla \mathbf{u}^{T}$ and $\mathbf{P}=\mathbf{I}-\mathbf{n} \otimes \mathbf{n}$. We assume pure advection here for the CIM concentration. Additionally we assume the jump conditions for velocity and flow stress tensor

$$
\begin{aligned}
{[\mathbf{u}]_{1}^{2} } & =0 \\
{[-p \mathbf{I}+\nu \mathbf{D}]_{1}^{2} \cdot \mathbf{n} } & =\mathbf{F}+\nabla_{\Gamma} \cdot(\lambda \mathbf{P}) \\
& =\mathbf{F}+\nabla_{\Gamma} \lambda-H \mathbf{n} \lambda
\end{aligned}
$$

where $\nabla_{\Gamma}=\mathbf{P} \nabla$ is the surface gradient and $\lambda$ is a Lagrange multiplier that provides the tension needed to enforce local inextensibility (Eq. (A-14)). This is as an analogue of the pressure in the momentum equation, which is used to 
enforce local incompressibility of the fluid. The surface force $\mathbf{F}$ is yet unspecified and will be chosen later such that thermodynamically consistency is assured. Note, that the RHS of Eq. A-17 corresponds to the singular forces introduced as RHS of the momentum equation in the diffuse interface case, Eq. (11). As shown in Appendix A.3, the time derivative of the curvature is

$$
\partial_{t} H=-\Delta_{\Gamma}(\mathbf{u} \cdot \mathbf{n})
$$

Using Eqs. A-12 $-\mathrm{A}-15$ and $\mathrm{A}-18$, the time derivative of the energy becomes

$$
\begin{gathered}
\partial_{t} E=\int_{\Gamma} b(c)\left(H-H_{0}(c)\right)\left(-\Delta_{\Gamma}+\mathbf{n} \cdot \nabla H\right)(\mathbf{u} \cdot \mathbf{n})+\frac{b(c)}{2}\left(H-H_{0}(c)\right)^{2} H \mathbf{u} \cdot \mathbf{n} \\
\quad-\frac{\partial E}{\partial c} \nabla c \cdot \mathbf{u} \mathrm{d} A+\int_{\Omega_{1} \cup \Omega_{2}}-\rho \mathbf{u} \cdot \nabla \mathbf{u} \cdot \mathbf{u}-\mathbf{u} \cdot \nabla p+\mathbf{u} \cdot \nabla \cdot(\nu \mathbf{D}) \mathrm{d} x
\end{gathered}
$$

Integration by parts ( 6 times) gives

$$
\begin{aligned}
\partial_{t} E=\int_{\Gamma} & -\Delta_{\Gamma}\left(b(c)\left(H-H_{0}(c)\right)\right) \mathbf{u} \cdot \mathbf{n}+b(c)\left(H-H_{0}(c)\right) \mathbf{n} \cdot \nabla H \mathbf{u} \cdot \mathbf{n} \\
& +\frac{b(c)}{2}\left(H-H_{0}(c)\right)^{2} H \mathbf{u} \cdot \mathbf{n} \\
& -\frac{\partial E}{\partial c} \nabla c \cdot \mathbf{u}+\mathbf{u} \cdot[-p \mathbf{I}+\nu \mathbf{D}]_{1}^{2} \cdot \mathbf{n} \mathrm{d} A-\int_{\Omega_{1} \cup \Omega_{2}} \frac{\nu}{2}|\mathbf{D}|^{2} \mathrm{~d} x .
\end{aligned}
$$

The boundary integrals on the outer domain boundary that arise from integration by parts vanish by assuming the boundary conditions of zero velocity, $\mathbf{u}=0$, or zero stress, $\mathbf{m} \cdot(\nu \mathbf{D}-p \mathbf{I})=0$, on $\delta \Omega$. Further, at the boundary of the membrane $\delta \Gamma$ we used normal pinning $(\mathbf{u} \cdot \mathbf{n}=0)$ and we assumed that at the boundary the membrane was flat curvature $(H=0)$ and that there were no CIMs $(c=0)$. Next observe that

$$
\int_{\Gamma} \mathbf{u} \cdot\left(\nabla_{\Gamma} \lambda-H \mathbf{n} \lambda\right)=\int_{\Gamma}-\lambda \nabla_{\Gamma} \cdot \mathbf{u}=0
$$

by assuming the boundary condition $\lambda=0$ on $\delta \Omega$. Using the above together with Eq. A-17 gives

$$
\begin{aligned}
\partial_{t} E=\int_{\Gamma} & -\Delta_{\Gamma}\left(b(c)\left(H-H_{0}(c)\right)\right) \mathbf{u} \cdot \mathbf{n}+b(c)\left(H-H_{0}(c)\right) \mathbf{n} \cdot \nabla H \mathbf{u} \cdot \mathbf{n}(\mathrm{A}-22) \\
& +\frac{b(c)}{2}\left(H-H_{0}(c)\right)^{2} H \mathbf{u} \cdot \mathbf{n}-\frac{\partial E}{\partial c} \nabla c \cdot \mathbf{u}+\mathbf{u} \cdot \mathbf{F} \mathrm{d} A-\int_{\Omega_{1} \cup \Omega_{2}} \frac{\nu}{2}|\mathbf{D}|^{2} \mathrm{~d} x .
\end{aligned}
$$

Furthermore, we compute

$\mathbf{n} \cdot \nabla H=\mathbf{n} \cdot \nabla \cdot\left(\nabla \mathbf{n}^{T}\right)=\nabla \cdot(\mathbf{n} \cdot \nabla \mathbf{n})-\nabla \mathbf{n}^{T}: \nabla \mathbf{n}=0-\nabla \mathbf{n}: \nabla \mathbf{n}=-\left\|\nabla_{\Gamma} \mathbf{n}\right\|^{2}$, 
where we have used that $H=\nabla \cdot \mathbf{n}$ and $\nabla \mathbf{n}^{T}=\nabla \mathbf{n}=\nabla_{\Gamma} \mathbf{n}$. Using Eq. A-23

$$
\begin{aligned}
\partial_{t} E=\int_{\Gamma} & -\Delta_{\Gamma}\left(b(c)\left(H-H_{0}(c)\right)\right) \mathbf{u} \cdot \mathbf{n}-b(c)\left(H-H_{0}(c)\right)\left\|\nabla_{\Gamma} \mathbf{n}\right\|^{2} \mathbf{u} \cdot \mathbf{n} \\
& +\frac{b(c)}{2}\left(H-H_{0}(c)\right)^{2} H \mathbf{u} \cdot \mathbf{n}-\frac{\partial E}{\partial c} \nabla c \cdot \mathbf{u}+\mathbf{u} \cdot \mathbf{F} \mathrm{d} A \\
& -\int_{\Omega_{1} \cup \Omega_{2}} \frac{\nu}{2}|\mathbf{D}|^{2} \mathrm{~d} x .
\end{aligned}
$$

The second law of thermodynamics requires the energy to be non-increasing, which can be accomplished with the choice of

$$
\begin{aligned}
\mathbf{F}= & \Delta_{\Gamma}\left(b(c)\left(H-H_{0}(c)\right)\right) \mathbf{n}+b(c)\left(H-H_{0}(c)\right)\left\|\nabla_{\Gamma} \mathbf{n}\right\|^{2} \mathbf{n} \\
& -\frac{b(c)}{2}\left(H-H_{0}(c)\right)^{2} H \mathbf{n}+\frac{\partial E}{\partial c} \nabla c .
\end{aligned}
$$

Hence, a thermodynamically consistent evolution of the membrane and the species concentration is given by Eqs. A-12 - A-15 with the stress jump condition

$$
\begin{aligned}
{[-p \mathbf{I}+\nu \mathbf{D}] \cdot \mathbf{n}=} & \nabla_{\Gamma} \lambda-H \mathbf{n} \lambda+\Delta_{\Gamma}\left(b(c)\left(H-H_{0}(c)\right)\right) \mathbf{n} \\
& +b(c)\left(H-H_{0}(c)\right)\left\|\nabla_{\Gamma} \mathbf{n}\right\|^{2} \mathbf{n} \\
& -\frac{b(c)}{2}\left(H-H_{0}(c)\right)^{2} H \mathbf{n}+\frac{\partial E}{\partial c} \nabla c
\end{aligned}
$$

This is consistent with the jump condition derived in [20], although we have presented a simpler derivation. Putting everything together, a thermodynamically consistent model for a CIM-covered membrane in viscous fluids is given by Eqs. A-12 $\mathrm{A}-15$ together with jump condition $\mathrm{A}-26$.

\section{A.3. Curvature time derivative}

We will now calculate the time derivative of the curvature $\partial_{t} H$ used in Section A.2. Note that this is not the advected time derivative (material derivative). Hence, for this expression to make sense we assume $\mathrm{H}$ to be extended off $\Gamma$, at least in a small neighborhood. The natural extension is such that $H(\mathbf{x})$ for $x \notin \Gamma$ is the curvature of a curve passing through $\mathrm{x}$ parallel to $\Gamma$. To compute $\partial_{t} H$ we introduce a phase field $\phi$ to represent the interface position. The normal is given by $\nabla \phi /|\nabla \phi|$ and hence

$$
\begin{aligned}
\partial_{t} H & =\partial_{t} \nabla \cdot \frac{\nabla \phi}{|\nabla \phi|}=\nabla \cdot \partial_{t} \frac{\nabla \phi}{|\nabla \phi|} \\
& =\nabla \cdot\left(\frac{\nabla \partial_{t} \phi}{|\nabla \phi|}-\frac{\nabla \phi \nabla \phi \cdot \nabla \partial_{t} \phi}{|\nabla \phi|^{3}}\right)=\nabla \cdot\left(\mathbf{P} \frac{\nabla \partial_{t} \phi}{|\nabla \phi|}\right) .
\end{aligned}
$$


Now, since $\Gamma$ (and hence also $\phi$ ) are advected: $\partial_{t} \phi=-\mathbf{u} \cdot \nabla \phi$. We obtain

$$
\begin{aligned}
\partial_{t} H & =-\nabla \cdot\left(\frac{\nabla_{\Gamma}(\mathbf{u} \cdot \nabla \phi)}{|\nabla \phi|}\right) \\
& =-\nabla \cdot\left(\nabla_{\Gamma}(\mathbf{u} \cdot \mathbf{n})+\mathbf{u} \cdot \nabla \phi \frac{\nabla_{\Gamma}|\nabla \phi|}{|\nabla \phi|^{2}}\right) \\
& =-\nabla \cdot\left(\nabla_{\Gamma}(\mathbf{u} \cdot \mathbf{n})\right)=-\Delta_{\Gamma}(\mathbf{u} \cdot \mathbf{n}),
\end{aligned}
$$

as claimed.

\section{References}

[1] S. Aland. Time integration for diffuse interface models for two-phase flow. J. Comp. Phys., 262C:58-71, 2014.

[2] S. Aland, S. Egerer, J. Lowengrub, and A. Voigt. Diffuse interface models of locally inextensible vesicles in a viscous fluid. J. Comp. Phys., 277:32-47, 2014.

[3] S. Aland and A. Voigt. Benchmark computations of diffuse interface models for two-dimensional bubble dynamics. Int. J. Num. Meth. Fluids, 69(3):747-761, 2012.

[4] J. Berro, V. Sirotkin, and T.D. Pollard. Mathematical modeling of endocytic actin patch kinetics in fission yeast: disassembly requires release of actin filament fragments. Mol. bio. cell, 21(16):2905-2915, 2010.

[5] Y. Chen, S.M. Wise, V.B. Shenoy, and J.S. Lowengrub. A Stable Scheme for a Nonlinear, Multispecies Tumor Growth Model with an Elastic Membrane. Int. J. Numer. Meth. Biomed. Engng., 30:726-754, 2014.

[6] C. Collins, J. Shen, and S.M. Wise. An Efficient, Energy Stable Scheme for the Cahn-Hilliard-Brinkman System. Commun. Comput. Phys., 13:929957, 2013.

[7] O. Daumke, A. Roux, and V. Haucke. BAR domain scaffolds in dynaminmediated membrane fission. Cell, 156:882-892, 2014.

[8] T A. Davis. Algorithm 832: UMFPACK V4.3 - an unsymmetric-pattern multifrontal method. ACM Trans. Math. Softw., 30(2):196-199, June 2004.

[9] A. Diegel, X. Feng, and S.M. Wise. Analysis of a Mixed Finite Element Method for a Cahn-Hilliard-Darcy-Stokes System. SIAM J. Numer. Anal., 53:127-152, 2015 .

[10] Q. Du, C. Liu, R. Ryham, and X.Q. Wang. Modeling the spontaneous curvature effects in static cell membrane deformations by a phase field formulation. Comm. Pure Appl. Anal., 4(3):537-548, 2005. 
[11] Q. Du, C. Liu, R. Ryham, and X.Q. Wang. A phase field formulation of the Willmore problem. Nonlinearity, 18:1249-1267, 2005.

[12] C.M. Elliott and B. Stinner. A surface phase field model for two-phase biological membranes. SIAM J. Appl. Math., 70:2904-2928, 2010.

[13] C.M. Elliott, B. Stinner, V. Styles, and R. Welford. Numerical computation of advection and diffusion on evolving diffuse interfaces. IMA J. Numer. Anal., 31:786-812, 2011.

[14] E.L. Elson, E. Fried, J.E. Dolbow, and G.M. Genin. Phase separation in biological membranes: Integration of theory and experiment. Ann. Rev. Biophys., 39:207-226, 2010.

[15] A. Embar, J. Dolbow, and E. Fried. Microdomain evolution on giant unilammellar vesicles. Biomech. Model. Mechanobiol., 12:597-615, 2013.

[16] M.L. Ferguson, K. Prasad, H. Boukari, D.L. Sackett, S. Krueger, E.M. Lafer, and R. Nossal. Clathrin Triskelia Show Evidence of Molecular Flexibility. Biophys J, 95(4):1945-1955, August 2008.

[17] C.M. Funkhouser, M. Mayer, F.J. Solis, and K. Thornton. Effects of interleaflet coupling on the morphologies of multicomponent lipid bilayer membranes. J. Chem. Phys., 138:024909, 2013.

[18] C.M. Funkhouser, F.J. Solis, and K. Thornton. Dynamics of two-phase lipid vesicles: Effects of mechanical properties on morphology evolution. Soft Matter, 6:3462-3466, 2010.

[19] C.M. Funkhouser, F.J. Solis, and K. Thornton. Dynamics of coarsening in multicomponent lipid vesicles with non-uniform mechanical properties. $J$. Chem. Phys., 140:144908, 2014.

[20] F. Haußer, W. Marth, S. Li, J. Lowengrub, A. Rätz, and A. Voigt. Thermodynamically consistent models for two-component vesicles. Int. J. Biomath. Biostat., 2:19-48, 2013.

[21] J. Hyman, H. Chen, P.P. Di Fiore, P. De Camilli, and A.T. Brunger. Epsin 1 undergoes nucleocytosolic shuttling and its eps15 interactor $\mathrm{NH}(2)$-terminal homology (ENTH) domain, structurally similar to Armadillo and HEAT repeats, interacts with the transcription factor promyelocytic leukemia Zn(2)+ finger protein (PLZF). J Cell Biol, 149(3):537-546, May 2000.

[22] A. J. Jin, K. Prasad, P. D. Smith, E. M. Lafer, and R. Nossal. Measuring the Elasticity of Clathrin-Coated Vesicles via Atomic Force Microscopy. Biophys. J., 90(9):3333-3344, May 2006.

[23] L. Johannes, R.G. Parton, P. Bassereau, and S. Mayor. Building endocytic pits without clathrin. Nat Rev Mol Cell Biol, pages 1-11, April 2015. 
[24] M. S. Lalonde and W. I. Sundquist. How hiv finds the door. PNAS, 109(46):18631-18632, 2012.

[25] J. Liu, Y. Sun, D. G. Drubin, and G. F. Oster. The mechanochemistry of endocytosis. PLoS biology, 7(9), 2009.

[26] J.S. Lowengrub, A. Raetz, and A. Voigt. Phase-field modeling of the dynamics of multicomponent vesicles: Spinodal decomposition, coarsening, budding and fission. Phys. Rev. E, 79:031926, 2009.

[27] K. Luby-Phelps. Cytoarchitecture and physical properties of cytoplasm: volume, viscosity, diffusion, intracellular surface area. Int. Rev. Cytol., 192:189-221, 2000.

[28] H.T. Mcmahon, M.M. Kozlov, and S. Martens. Membrane Curvature in Synaptic Vesicle Fusion and Beyond. Cell, 140(5):601-605, March 2010.

[29] S. A. Nowak and T. Chou. Membrane lipid segregation in endocytosis. Phys Rev. E, 78(2):021908, 2008.

[30] B. Peleg, A. Disanza, G. Scita, and N. Gov. Propagating Cell-Membrane Waves Driven by Curved Activators of Actin Polymerization. PLoS ONE, 6(4):e18635, April 2011.

[31] A. Rätz and A. Voigt. PDE's on surfaces - a diffuse interface approach. Comm. Math. Sci., 4:575-590, 2006.

[32] P. J. Robinson. How to fill a synapse. Science, 316(5824):551-553, 2007.

[33] H. Shen, M. Pirruccello, and P. De Camilli. Snapshot: membrane curvature sensors and generators. Cell, 150(6), 2012.

[34] J.S. Sohn, S. Li, X. Li, and J.S. Lowengrub. Axisymmetric multicomponent vesicles: A comparison of hydrodynamic and geometric models. Int. J. Num. Meth Biomed. Engng., pages 346-368, 2012.

[35] J.S. Sohn, Y.H. Tseng, S. Li, A. Voigt, and J. Lowengrub. Dynamics of multicomponent vesicles in a viscous fluid. J. Comp. Phys., 229:119-144, 2010.

[36] J. C. Stachowiak, F. M. Brodsky, and E. A. Miller. A cost-benefit analysis of the physical mechanisms of membrane curvature. Nat. Cell Biol., 15(9):1019-1027, 2013.

[37] K.E. Teigen, X. Li, J. Lowengrub, F. Wang, and A. Voigt. A diffuse-interface approach for modeling transport, diffusion and adsorption/desorption of material quantities on a deforming interface. Comm. Math. Sci., 7:1009-1037, 2009. 
[38] K.E. Teigen, P. Song, J. Lowengrub, and A. Voigt. A diffuse-interface method for two-phase flows with soluble surfactants. J. Comput. Phys., 230:375, 2011.

[39] S. Vey and A. Voigt. Amdis: adaptive multidimensional simulations. Comp. Vis. Sci., 10(1):57-67, March 2007.

[40] X. Wang and Q. Du. Modelling and simulations of multi-component lipid membranes and open membranes via diffuse interface approaches. J. Math. Biol., 56:347-371, 2008.

[41] S. Watanabe, B. R. Rost, M. Camacho-Pérez, M. W. Davis, B. SöhlKielczynski, C. Rosenmund, and E. M. Jorgensen. Ultrafast endocytosis at mouse hippocampal synapses. Nature, 504(7479):242-247, 2013.

[42] D. Wirtz. Particle-Tracking Microrheology of Living Cells: Principles and Applications. Ann. Rev. Biophys., 38(1):301-326, June 2009.

[43] S.M. Wise. Unconditionally stable finite difference, nonlinear multigrid simulation of the Cahn-Hilliard-Hele-Shaw system of equations. J. Sci. Comput., 44:38-68, 2010.

[44] Y. Zhao and Q. Du. Diffuse interface model of multicomponent vesicle adhesion and fusion. Phys. Rev. E, 84(1):011903, July 2011. 